\title{
Comparison of developed FLC and P\&O MPPT algorithms for improving PV system performance at variable irradiance conditions
}

\begin{abstract}
Purpose: This work aims to overcome the drawbacks of the nonlinear characteristics of the photo-voltaic (PV) system which are affected by the atmospheric variations.

Design/methodology/approach: As a result, the optimum power point on these characteristics accordingly changes and the efficiency of photovoltaic systems reduces. Maximum power point tracking (MPPT) algorithms track this optimum point and enhance the efficiency despite the irradiance and temperature changes.

Findings: The conventional perturbation and observation $(\mathrm{P} \& \mathrm{O})$ algorithm uses fixed step sizes to increment and decrement the duty ratio that leads to slow response time and continuous oscillation around the MPP at steady state conditions. The paper proposes a fuzzy logic-based controller that overcomes the drawbacks of $\mathrm{P} \& \mathrm{O}$ algorithm in term of response time and the oscillation.
\end{abstract}

Originality/value: MATLAB/Simulink environment was used to model and simulate the KC200GT PV module, direct current (DC)-DC boost converter and the MPPT algorithms.

Keyword: $\quad$ Boost converter; FLC; MPPT; P\&O; Photovoltaic system 